\title{
Effect of Sputtering Power on the Structural and Optical Properties of Inn Nanodots on $\mathrm{Al}_{2} \mathrm{O}_{3}$ by Magnetron Sputtering
}

\author{
Ziming Zhang ${ }^{a}$, Jingjie Li ${ }^{a}$, Yijian Zhou ${ }^{a}$, Hongyuan Fu ${ }^{a}$, Zixu Zhang ${ }^{a}$, Guojiao Xiang ${ }^{a}$, \\ Yang Zhao $^{a * \mathbb{D}, \text { Shiwei Zhuang }}{ }^{b}$, Fan Yang ${ }^{c}$, and Hui Wang ${ }^{a}$ \\ ${ }^{a}$ Henan University of Science and Technology, School of Physics and Engineering, Henan Key Laboratory \\ of Photoelectric Energy Storage Materials and Applications, 471003, Luoyang, China \\ ${ }^{b}$ Zhengzhou University, Department of Physics and Engineering, Key Laboratory of Materials Physics \\ of Ministry of Education, 450052, Zhengzhou, China \\ 'Jilin Jianzhu University, School of Electrical Engineering and Computer, Jilin Provincial Key Laboratory \\ of Architectural Electricity \& Comprehensive Energy Saving, 130118, Changchun, China
}

Received: June 13, 2019; Revised: August 31, 2019; Accepted: November 25, 2019

In this work, we reported the effects of sputtering power on the structure, optical and electrical properties of $\mathrm{InN}$ nanodots prepared on $\mathrm{A} 12 \mathrm{O} 3$ substrate by magnetron sputtering. The results showed that the as-grown InN films exhibited uniform nanodot morphology and the size of the InN nano grains increased with the sputtering power was increased. The InN nanodot exhibited highly c-axis prefered orientation with mainly InN (002) diffraction. The optical band gap of InN samples showed an decreasing trend with the increase in sputteirng power. Moreover, the electrical properties of the InN samples were discussed in detail by hall effect and the carrier concentration and mobility could be adjusted from $3.233 \times 1019$ to $1.655 \times 1020 \mathrm{~cm}-3$ and 1.151 to $10.101 \mathrm{~cm} 2 / v^{\bullet} \mathrm{s}$, respectively. These results will lay a good fundation for the applicaion of InN material in the field of gas sensers and light emitting diodes.

Keywords: InN nanodots, magnetron sputtering, highly preferred orientation, electrical characteristic.

\section{Introduction}

Indium nitride, as a kind of novel material among the III-V group nitrides, has been broadly applied in the fields of high-speed electronic devices, high efficiency solar cells, infrared light emitting diodes and laser diodes ${ }^{1-4}$. It has lower effective electron mass, higher saturation electron drift rate and higher electron mobility, which make it ideal for the development of the above optoelectronic devices ${ }^{5-7}$. However, the growth of high-quality $\mathrm{InN}$ films is difficult due to the lower decomposition temperature of $\operatorname{InN}\left(\sim 550^{\circ} \mathrm{C}\right)^{8}$. So far, InN films could be prepared by using a variety of techniques. It has been confirmed that the bandgap of single crystal InN grown by molecular beam epitaxy (MBE) or metal organic vapor phase epitaxy (MOVPE) is around $0.7 \mathrm{eV}^{9}$. It is noted that the initial band gap value of the InN films prepared by magnetron sputtering is about 2.0 $\mathrm{eV}$ due to the Burstein-Moss effect and oxygen impurity incorporation ${ }^{10}$. Nevertheless, from the point of experiment cost and low temperature of growth condition, the sputtering has the advantages over the other methods such as MBE or MOVPE. At the same time, the material prepared by magnetron sputtering usually exhibited highly textured structure like nanodots, nanowires and nanopillars, which would be affected relatively lighter by the lattice mismatch between the epilayer and substrate. This would be benefit for the application of InN-based gas sensers and photodevices.

*e-mail: 1wc9442@126.com
Recently, Chen et al. have reported the synthesis of selforganized InN nanodots on $\mathrm{Si}$ substrate by droplet epitaxy method $^{11}$. Li et al. have reported the growth of well-aligned InN nanorods on glass substrate by metal-organic chemical vapor deposition ${ }^{12}$. In this work, we reported the growth of well-oriented InN nanodots on sapphire substrate by magnetron sputtering. The structure, morphology, optical and electrical characteristics of $\mathrm{InN}$ nanodots were systematic investigated as a function of the sputtering power.

\section{Experiments}

InN films were prepared on sapphire substrate by radio frequency (RF) vacuum magnetron sputtering system. The substrate was cleaned by ultrasonic cleaning in acetone $(\mathrm{CH} 3 \mathrm{COCH} 3)$, alcohol $(\mathrm{C} 2 \mathrm{H} 5 \mathrm{OH})$ and deionized water respectively for 5 minutes. Then the substrates were dried with nitrogen and placed $5 \mathrm{~cm}$ from the target in the reaction chamber. The diameter and thickness of the highly purity (99.999\%) In target was 50 and $3 \mathrm{~mm}$. The substrate temperature and pressure were kept at $200{ }^{\circ} \mathrm{C}$ and 1 pa, respectively. The nitrogen flux added into the chamber were maintained at $20 \mathrm{sccm}$. Before formal sputtering, the target was under presputter for 5 minutes to remove the contaminants on the target surface. Then the sputtering was controlled at 1 hour. Under the above experimental conditions, InN samples marked A-D were prepared by setting the sputtering power at $80,90,100$ and $110 \mathrm{~W}$, respectively. 
The crystallization of $\mathrm{InN}$ samples were analyzed by X-ray diffraction (XRD; D8 Discover Gadds) measurement. The surface morphology of InN samples were investigated by atomic force microscope (AFM; Veeco Dimension 3100 ). Moreover, the absorption and electrical characteristics of InN samples were analyzed by UV-2700 spectrophotometer and Accent HL5500PC Hall effect system, respectively.

\section{Results and Discussion}

To analyze the crystal structure of $\mathrm{InN}$ films prepared under different sputtering power, XRD measurements were performed as shown in Fig. 1. As seen from Fig. 1, all InN samples exhibited mainly InN (002) diffraction, which indicated that all InN samples grew preferentially along the $\mathrm{c}$-axis direction and the (002) orientation. Besides, the intensity of the InN (002) diffractions increased with the sputtering power was increased from 80 to $110 \mathrm{~W}$. And the full width at half maximum (FWHM) of InN (002) diffraction showed a decreasing trend as the sputtering power was increased. These results indicated that the increase of sputtering power is beneficial to the growth and crystallization of $\mathrm{InN}$ grains ${ }^{6}$. This was probobaly attributed to the increase of kinetic energy for InN grains to nucleation as increasing the sputtering power ${ }^{13}$. In addition, relatively weak InN (101) diffraction peaked at $\sim 32.5^{\circ}$ was also detected. It was noted that the intensity of $\mathrm{InN}$ (101) diffraction changed little with the increase of sputtering power. While the intensity ratio of InN $(002) /(101)$ for the InN samples grown at sputtering power of $80,90,100$ and $110 \mathrm{~W}$ was $3.1,5.2,10.6$ and 19.8 , respectively. This indicated that the sputtering power was good for the InN (002) preferential growth orientation. On the other hand, the InN films grown by MBE usually exhibited only InN (002) and (004) orientation ${ }^{14}$, which was different from our results. This was usually due to the fact that the materials prepared by sputtering usually exhibited polycrystal qualities ${ }^{15}$.

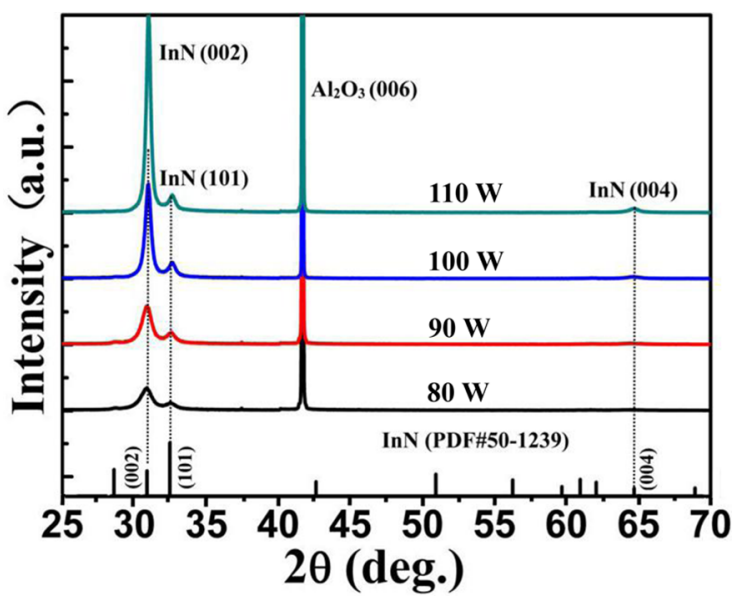

Figure 1. w-2 $\theta$ XRD scans of InN samples deposited on sapphire substrates at different sputtering powers.
The atomic force microscope (AFM) images of the InN samples grown at $80,90,100$ and $110 \mathrm{~W}$ were shown in Fig. 2(a), Fig. 2(b), Fig. 2(c) and Fig. 2(d), respectively. As seen from Figure 2, it is evident that the sputtering power has a critical effect on the surface morphologies of InN samples. This also can be seen from the SEM images of the InN samples as shown in Fig. 3. By comparing the morphology images of four samples, it can be seen that all samples are composed of InN nanodots, and the size of InN nanodots increased as the sputtering power was increased from 80 to $100 \mathrm{~W}$. This result was usually attributed to the increase in sputtering rate as the sputtering power was increased ${ }^{16}$. However, the nanodot surface deteriorated when the sputtering power was added to $110 \mathrm{~W}$. This phenomenon was similar with Zhao's et al. Report ${ }^{13}$, which was usually due to the decrease of the kinetic energy of the sputtered InN particles caused by secondary sputtering when the sputtering power was excessively high. Moreover, the surface roughness of InN nanodots could be calculated from $5 \times 5$ um 2 AFM scans according to analysis from the NanoScrope Analysis software and the roughness value for $\mathrm{InN}$ samples grown at the sputtering power of $80,90,100$ and $110 \mathrm{~W}$ were 1.59 , $1.66,2.22$ and $3.13 \mathrm{~nm}$, respectively.

The optical properties of InN samples were determined by optical absorption measurement. These experimental results were then combined with theoretical calculations. The value of optical band gap could be determined following equation derive independently by Tauc et al ${ }^{17}$ :

$$
\alpha h v=A\left(h v-E_{g}\right)^{1 / 2}
$$

Where $\mathrm{A}$ is a constant and hv is the photon energy. The value of the Eg can be calculated by the extrapolation of linear part to the horizontal axis as shown in Fig. 4. The Eg for the InN samples marked A-D sputtered at the power of $80,90,100$, and $110 \mathrm{~W}$, respectively, was found to be 1.83 , $1.82,1.78$ and $1.77 \mathrm{ev}$, respectively. These values of bandgap indicated that the as-grown InN materials could be used in the filed of near infrared photodetectors and light emitting diodes 18, 5 . Moreover, the value of the bandgap was in according with the reported results (1.60-1.90 eV) obtained by Felip et al. using the radio frequency sputtering ${ }^{19}$. However, the results was more larger than the established bandgap values of 0.7-1.0 eV reported for high quality single-crystalline InN grown by MBE or MOVPE. The larger optical bandgap could be attributed to the high free electron concentration, which usually induced the Burstein-Moss effect ${ }^{20}$.

Fig. 5 shows the relationship between the electrical properties of InN samples and the sputtering power. As can be seen from Fig. 5, all InN samples exhibited strong n-type conductivity characteristics with high carrier concentrations. And the carrier concentration and mobility could be adjusted from $3.233 \times 1019$ to $1.655 \times 1020 \mathrm{~cm}-3$ and 1.151 to $10.101 \mathrm{~cm} 2 / \mathrm{v} \bullet \mathrm{s}$, respectively. 

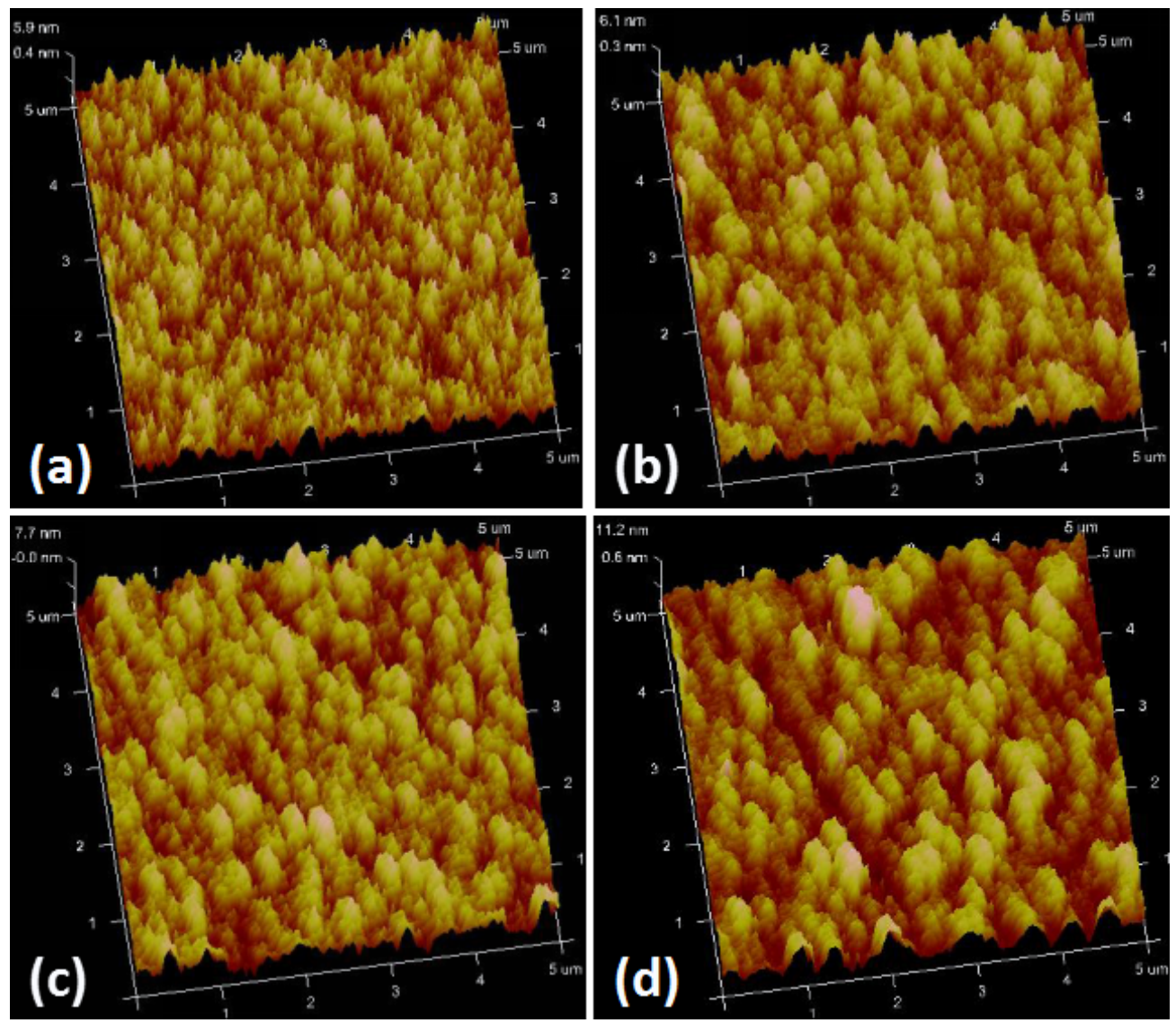

Figure 2. 3D morphology of the AFM images $(5 \times 5$ um2) of the InN nanodots grown at different sputtering powers: (a) $80 \mathrm{~W}$, (b) $90 \mathrm{~W}$, (c) $100 \mathrm{~W}$ and (d) $110 \mathrm{~W}$.
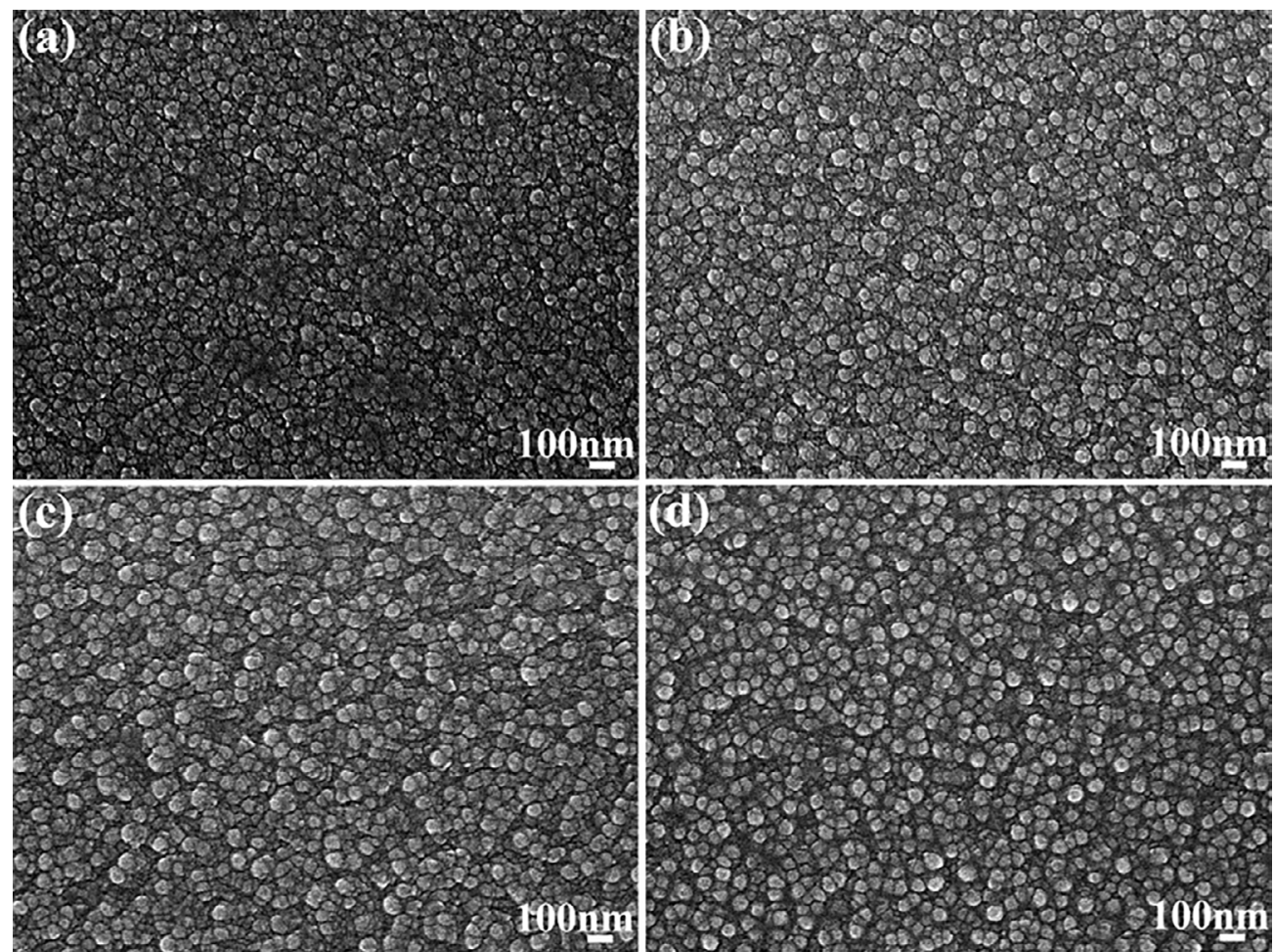

Figure 3. SEM images of the InN samples grown at different sputtering powers: (a) $80 \mathrm{~W}$, (b) $90 \mathrm{~W}$, (c) $100 \mathrm{~W}$ and (d) $110 \mathrm{~W}$. 
These values were in good agreement with previous results reported in Wang's et al. research work ${ }^{6}$. One can observe that the mobility of the InN samples were significantly increased with the increase in sputtering power, which was mainly attributed to the increased surface migration energy of the adatoms. Besides, the resistivities of the $\mathrm{InN}$ samples exhibited an increasing trend when the sputtering power was increased as shown in Fig. 5(b). This was in according with the trend of surface roughness, which was mainly due to the influence of the changes in InN crystal qualities ${ }^{14}$. These results indicated that the electrical properties of $\mathrm{InN}$ nanodots can be improved by the selection of appropriate sputtering power.

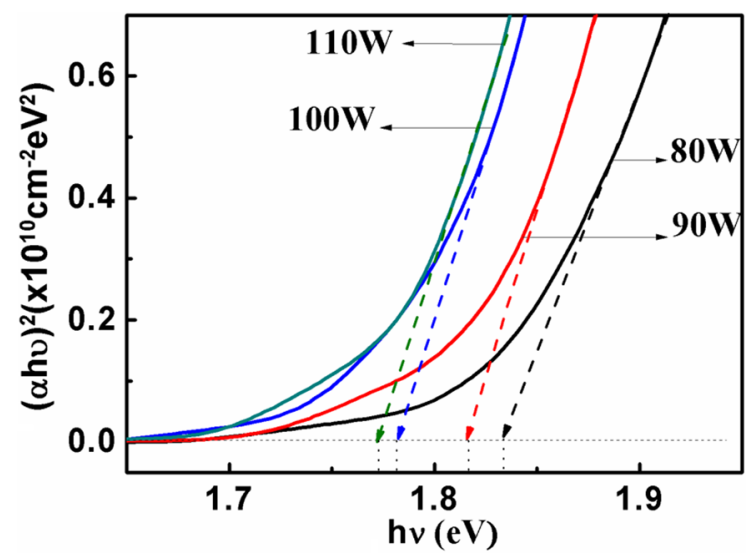

Figure 4. Optical absorption spectra of InN samples deposited on sapphire substrates at different sputtering power: $80 \mathrm{~W}, 90 \mathrm{~W}$, $100 \mathrm{~W}$ and $100 \mathrm{~W}$.

\section{Conclusion}

InN nanodots were prepared by magnetron sputtering on sapphire substrates at various sputtering powers. We have presented a detailed research on the influence of sputtering power on the physical properties of InN nanodots. It was found that the InN nanodots grew preferentially along the c-axis direction with InN (002) orientation. It had a homogeneous surface and the size of InN nanodots increased as the sputtering power was increased. Moreover, the optical band gap of the InN nanodots were found to be around $1.77-1.83 \mathrm{eV}$. And the Hall test results showed that all $\mathrm{InN}$ nanodots exhibited n-type conductivity characteristics with higher carrier concentration. It was believed that the $\mathrm{InN}$ nanodots can be used as a good n-type semiconductor material in the field of photovoltaic devices.

\section{Acknowledgment}

This work was supported by the National Natural Science Foundation of China (Grant Nos. 61674052 and 11404097), the Key Scientific Research Projects of Higher Education Institutions of Henan Provinc, China (Grant No. 20A140012), the Innovation and Entrepreneurship Training Program for Provincial Undergraduates of Henan Province (Grant No. S201910464024), the Student Research Training Program of Henan University of Science and Technology (Grant No. 2019208), and the Student Research Training Program of School of Physics and Engineering of Henan University of Science and Technology (Grant Nos. WLSRTP201910 and WLSRTP201802).

\section{(b)}
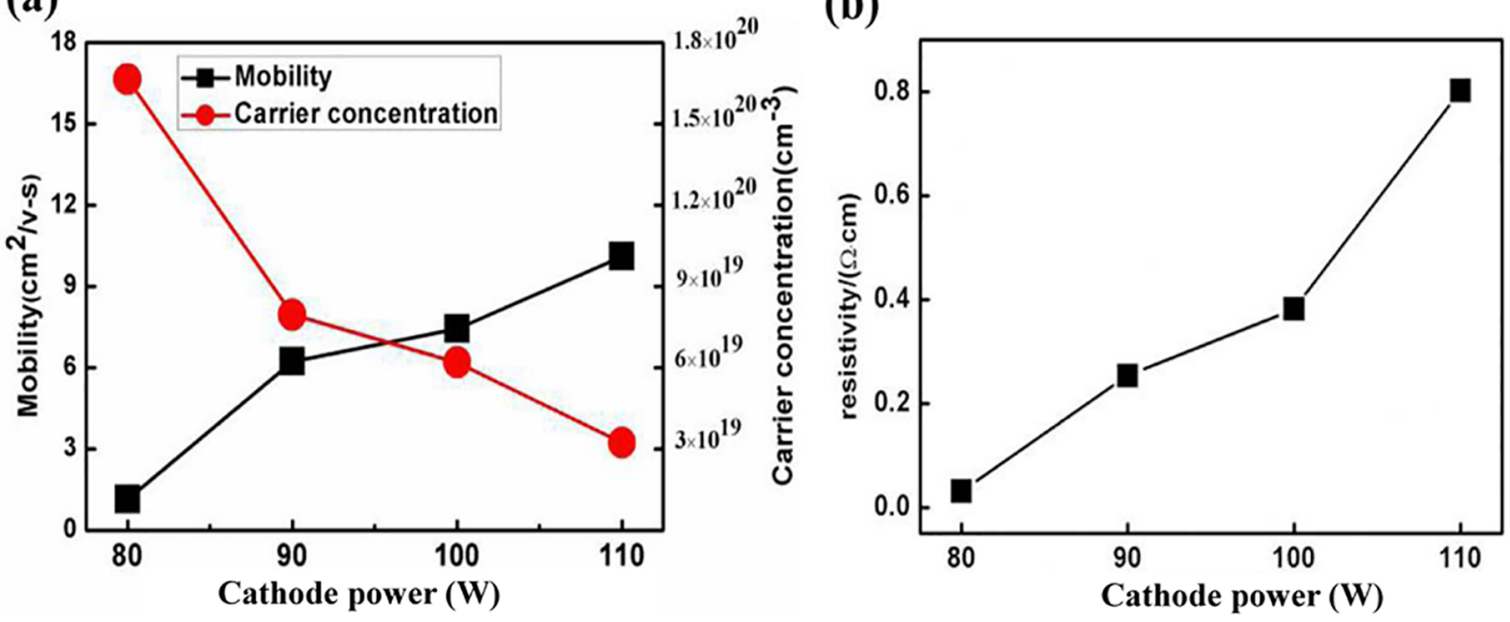

Figure 5. (a) Hall mobilities and carrier concentrations of $\mathrm{InN}$ samples deposited on sapphire substrates at different sputtering powers, (b) Resistivities of InN samples at different sputtering powers. 


\section{Reference}

1. Reilly CE, Lund C, Nakamura S, Mishra UK, DenBaars SP, Keller $\mathrm{S}$. Infrared luminescence from $\mathrm{N}$-polar $\mathrm{InN}$ quantum dots and thin films grown by metal organic chemical vapor deposition. Applied Physics Letters. 2019;114(24):241103.

2. Zhao Y, Wang H, Li XZ, Li JJ, Shi ZF, Wu GG, et al. Parametric study on the well-oriented growth of InxAll-xN nanodots by magnetron sputtering. Materials Science in Semiconductor Processing. 2019;102:104583.

3. Shi ZF, Li Y, Zhang YT, Chen YS, Li XJ, Wu D, et al. Nano Letters. 2017;17:313-21.

4. Zhao Y, Wang H, Gong XY, Li QZ, Wu GG, Li WC, et al. Near infrared electroluminescence from $\mathrm{p}-\mathrm{NiO} / \mathrm{n}-\mathrm{InN} / \mathrm{n}-\mathrm{GaN}$ lightemitting diode fabricated by PAMBE. Journal of Luminescence. 2017;186:243-6.

5. Wang H, Zhao Y, Li XZ, Zhen ZQ, Li QZ, Li HH, et al. Dominant near infrared light-emitting diodes based on $\mathrm{p}-\mathrm{NiO} / \mathrm{n}-\mathrm{InN}$ heterostructure on $\mathrm{SiC}$ substrate. Journal of Alloys and Compounds. 2018;735:1402-5.

6. Wang H, Zhao Y, Li Z, Li JJ, Zhang ZM, Wan S, et al. Growth of well-oriented InN nanodots by magnetron sputtering with varying sputtering temperature. Journal of Vacuum Science and Technology: B. 2018;36:041204.

7. Shi ZF, Li Y, Li S, Li XY, Wu D, Xu TT, et al. Localized surface plasmon enhanced all-inorganic perovskite quantum dot lightemitting diodes based on coaxial core/shell heterojunction architecture. Advanced Functional Materials. 2018;28(20):1707031.

8. Koukitu A, Takahashi N, Seki H. Thermodynamic study on metalorganic vapor-phase epitaxial growth of group III nitrides. Japanese Journal of Applied Physics. 1997;36(Pt 2):L1136.

9. Bhuiyan AG, Hashimoto A, Yamamoto A. Indium nitride ( $\mathrm{InN})$ : a review on growth, characterization, and properties. Journal of Applied Physics. 2003;94(5):2779.
10. Wu J, Walukiewicz W, Shan W, Yu KM, Ager JW, Haller EE, et al. Effects of the narrow band gap on the properties of $\mathrm{InN}$. Physical Review: B. 2002;66(20):201403.

11. Chen HJ, Yang D, Huang TW, Yu IS. Formation and temperature effect of inn nanodots by PA-MBE via droplet epitaxy technique. Nanoscale Research Letters. 2016;11:241.

12. Li HJ, Zhao GJ, Wei HY, Wang LS, Chen Z, Yang SY. Growth of well-aligned InN nanorods on amorphous glass substrates. Nanoscale Research Letters. 2016;11(1):270.

13. Zhao Y, Wang H, Yang F, Wang ZY, Li JJ, Gao YT, et al. Materials Research-Ibero-American Journal. 2018;21:e20170836.

14. Wang H, Zhao Y, Li XZ, Zhen ZQ, Li HH, Wang JG, et al. Vacuum. 2017;144:199-202.

15. Wu J, Walukiewicz W, Li SX, Armitage R, Ho JC, Weber ER. Effects of electron concentration on the optical absorption edge of InN. Applied Physics Letters. 2004;84:2805.

16. Biju KP, Jain MK. The effect of RF power on the growth of InN films by modified activated reactive evaporation. Applied Surface Science. 2008;254(22):7259-65.

17. Tauc J, Grigorovici R, Vancu A. Optical properties and electronic structure of amorphous germanium. Physica Status Solidi: B. 1996;15(2):627.

18. Chowdhury AM, Pant R, Roul B, Singh DK, Nanda KK, Krupanidhi SB. Double Gaussian distribution of barrier heights and self-powered infrared photoresponse of InN/AIN/Si (111) heterostructure. Journal of Applied Physics. 2019;126(2):025301.

19. Valdueza-Felip S, Naranjo FB, González-Herráez M, Lahourcade L, Monroy E, Fernandez S. Influence of deposition conditions on nanocrystalline $\mathrm{InN}$ layers synthesized on $\operatorname{Si}\left(\begin{array}{lll}1 & 1 & 1\end{array}\right)$ and $\mathrm{GaN}$ templates by RF sputtering. Journal of Crystal Growth. 2010;312(19):2689-94.

20. Kumar M, Bhat TN, Rajpalke MK, Roul B, Kalghatgi AT, Krupanidhi $\mathrm{SB}$. Indium flux, growth temperature and RF power induced effects in $\mathrm{InN}$ layers grown on $\mathrm{GaN} / \mathrm{Si}$ substrate by plasmaassisted MBE. Journal of Alloys and Compounds. 2012;513:6-9. 